\title{
Research Article \\ Fixed Point and Asymptotic Analysis of Cellular Neural Networks
}

\author{
Xianghong Lai ${ }^{1}$ and Yutian Zhang ${ }^{2}$ \\ ${ }^{1}$ School of Economics \& Management, Nanjing University of Information Science E Technology, \\ Nanjing 210044, China \\ ${ }^{2}$ School of Mathematics $\mathcal{E}$ Statistics, Nanjing University of Information Science and Technology, \\ Nanjing 210044, China
}

Correspondence should be addressed to Yutian Zhang, ytzhang81@163.com

Received 24 April 2012; Revised 16 July 2012; Accepted 2 August 2012

Academic Editor: Naseer Shahzad

Copyright (C) 2012 X. Lai and Y. Zhang. This is an open access article distributed under the Creative Commons Attribution License, which permits unrestricted use, distribution, and reproduction in any medium, provided the original work is properly cited.

We firstly employ the fixed point theory to study the stability of cellular neural networks without delays and with time-varying delays. Some novel and concise sufficient conditions are given to ensure the existence and uniqueness of solution and the asymptotic stability of trivial equilibrium at the same time. Moreover, these conditions are easily checked and do not require the differentiability of delays.

\section{Introduction}

Cellular neural networks (CNNs) were firstly proposed by Chua and Yang in 1988 [1, 2] and have become a research focus for their numerous successful applications in various fields such as optimization, linear, and nonlinear programming, associative memory, pattern recognition, and computer vision. Owing to the finite switching speed of neurons and amplifiers in the implementation of neural networks, it turns out that the time delays are inevitable and therefore the model of delayed cellular neural networks (DCNNs) is of greater realistic significance. Research on the dynamic behaviors of CNNs and DCNNs has received much attention, and nowadays there have been a large number of achievements reported [3-5].

In fact, besides delay effects, stochastic and impulsive as well as diffusing effects are also likely to exist in the neural networks. As a result, they have formed complex CNNs including impulsive delayed reaction-diffusion CNNs, stochastic delayed reaction-diffusion CNNs, and so forth. One can refer to [6-11] for the relevant researches. Synthesizing the existing publications about complex CNNs, we find that Lyapunov method is the primary 
technique. However, we also notice that there exist lots of difficulties in the applications of corresponding results to practical problems and so it does seem that new methods are needed to address those difficulties.

Encouragingly, Burton and other authors have recently applied the fixed point theory to investigate the stability of deterministic systems and obtained more applicable results, for example, see the monograph [12] and the papers [13-24]. Furthermore, there has been found that the fixed point theory is also effective to the stability analysis of stochastic (delayed) differential equations, see [25-31]. Particularly, in [26-28], Luo used the fixed point theory to study the exponential stability of mild solutions of stochastic partial differential equations with bounded delays and with infinite delays. In [29, 30], Sakthivel and Luo used the fixed point theory to investigate the asymptotic stability in $p$ th moment of mild solutions to nonlinear impulsive stochastic partial differential equations with bounded delays and with infinite delays. In [31], Luo used the fixed point theory to study the exponential stability of stochastic Volterra-Levin equations. With these motivations, we wonder if we can use the fixed point theory to study the stability of complex neural networks, thus obtaining more applicable results.

In the present paper, we aim to discuss the asymptotic stability of CNNs and DCNNs. Our method is based on the contraction mapping theory, which is different from the usual method of Lyapunov theory. Some new and easily checked algebraic criteria are presented ensuring the existence and uniqueness of solution and the asymptotic stability of trivial equilibrium at the same time. These sufficient conditions do not require even the differentiability of delays, let alone the monotone decreasing behavior of delays.

\section{Preliminaries}

Let $\mathrm{R}^{n}$ denote the $n$-dimensional Euclidean space and $\|\cdot\|$ represent the Euclidean norm. $\mathcal{N} \triangleq\{1,2, \ldots, n\} . R_{+}=[0, \infty) . C(X, Y)$ corresponds to the space of continuous mappings from the topological space $X$ to the topological space $Y$.

In this paper, we consider the cellular neural network described by

$$
\begin{gathered}
\frac{\mathrm{d} x_{i}(t)}{\mathrm{d} t}=-a_{i} x_{i}(t)+\sum_{j=1}^{n} b_{i j} f_{j}\left(x_{j}(t)\right), \quad t \geq 0, \\
x_{i}(0)=x_{0 i}
\end{gathered}
$$

and the following cellular neural network with time-varying delays as

$$
\begin{gathered}
\frac{\mathrm{d} x_{i}(t)}{\mathrm{d} t}=-a_{i} x_{i}(t)+\sum_{j=1}^{n} b_{i j} f_{j}\left(x_{j}(t)\right)+\sum_{j=1}^{n} c_{i j} g_{j}\left(x_{j}\left(t-\tau_{j}(t)\right)\right), \quad t \geq 0 \\
x_{i}(s)=\varphi_{i}(s), \quad-\tau \leq s \leq 0
\end{gathered}
$$

where $i \in \mathcal{N}$ and $n$ is the number of neurons in the neural network. $x_{i}(t)$ corresponds to the state of the $i$ th neuron at time $t . \mathbf{x}_{0}=\left(x_{01}, \ldots, x_{0 n}\right)^{\mathrm{T}} \in \mathrm{R}^{n} \cdot f_{j}\left(x_{j}(t)\right)$ denotes the activation function of the $j$ th neuron at time $t$ and $g_{j}\left(x_{j}\left(t-\tau_{j}(t)\right)\right)$ is the activation function of the $j$ th 
neuron at time $t-\tau_{j}(t)$. The constant $b_{i j}$ represents the connection weight of the $j$ th neuron on the $i$ th neuron at time $t$. The constant $a_{i}>0$ represents the rate with which the $i$ th neuron will reset its potential to the resting state when disconnected from the network and external inputs. The constant $c_{i j}$ represents the connection strength of the $j$ th neuron on the $i$ th neuron at time $t-\tau_{j}(t)$, where $\tau_{j}(t)$ corresponds to the transmission delay along the axon of the $j$ th neuron and satisfies $0 \leq \tau_{j}(t) \leq \tau$ ( $\tau$ is a constant). $f_{j}(\cdot), g_{j}(\cdot) \in C(\mathrm{R}, \mathrm{R})$. $\varphi(s)=\left(\varphi_{1}(s), \ldots, \varphi_{n}(s)\right)^{\mathrm{T}} \in \mathrm{R}^{n}$ and $\varphi_{i}(s) \in C([-\tau, 0], \mathrm{R})$. Denote $|\varphi|=\sup _{s \in[-\tau, 0]}\|\varphi(s)\|$.

Throughout this paper, we always assume that $f_{j}(0)=g_{j}(0)=0$ for $j \in \mathcal{N}$ and therefore (2.1) and (2.3) admit a trivial equilibrium $\mathbf{x}=0$.

Denote by $\mathbf{x}\left(t ; 0, \mathbf{x}_{0}\right)=\left(x_{1}\left(t ; 0, x_{01}\right), \ldots, x_{n}\left(t ; 0, x_{0 n}\right)\right)^{\mathrm{T}} \in \mathrm{R}^{n}$ the solution of (2.1) with the initial condition (2.2) and denote by $\mathbf{x}(t ; s, \varphi)=\left(x_{1}\left(t ; s, \varphi_{1}\right), \ldots, x_{n}\left(t ; s, \varphi_{n}\right)\right)^{\mathrm{T}} \in \mathrm{R}^{n}$ the solution of (2.3) with the initial condition (2.4).

Definition 2.1 (see [32]). The trivial equilibrium $\mathbf{x}=0$ of (2.1) is said to be stable if for any $\varepsilon>0$, there exists $\delta>0$ such that for any initial condition $\mathbf{x}_{0}$ satisfying $\left\|\mathbf{x}_{0}\right\|<\delta$,

$$
\left\|\mathbf{x}\left(t ; 0, \mathbf{x}_{0}\right)\right\|<\varepsilon, \quad t \geq 0 .
$$

Definition 2.2 (see [32]). The trivial equilibrium $\mathbf{x}=0$ of (2.1) is said to be asymptotically stable if it is stable and for any $\mathbf{x}_{0} \in \mathrm{R}^{n}$,

$$
\lim _{t \rightarrow \infty}\left\|\mathbf{x}\left(t ; 0, \mathbf{x}_{0}\right)\right\|=0
$$

Definition 2.3 (see [32]). The trivial equilibrium $\mathbf{x}=0$ of (2.3) is said to be stable if for any $\varepsilon>0$, there exists $\delta>0$ such that for any initial condition $\varphi(s) \in C\left([-\tau, 0], \mathrm{R}^{n}\right)$ satisfying $|\varphi|<\delta$,

$$
\|\mathbf{x}(t ; s, \varphi)\|<\varepsilon, \quad t \geq 0
$$

Definition 2.4 (see [32]). The trivial equilibrium $\mathbf{x}=0$ of (2.3) is said to be asymptotically stable if it is stable and for any initial condition $\varphi(s) \in C\left([-\tau, 0], \mathrm{R}^{n}\right)$,

$$
\lim _{t \rightarrow \infty}\|\mathbf{x}(t ; s, \varphi)\|=0
$$

The consideration of this paper is based on the following fixed point theorem.

Lemma 2.5 (see [33]). Let $\Upsilon$ be a contraction operator on a complete metric space $\Theta$, then there exists a unique point $\zeta \in \Theta$ for which $\Upsilon(\zeta)=\zeta$.

\section{Asymptotic Stability of Cellular Neural Networks}

In this section, we will simultaneously consider the existence and uniqueness of solution to (2.1)-(2.2) and the asymptotic stability of trivial equilibrium $\mathbf{x}=0$ of (2.1) by means 
of the contraction mapping principle. Before proceeding, we firstly introduce the following assumption:

(A1) There exist nonnegative constants $l_{j}$ such that for $\eta, v \in \mathrm{R}$,

$$
\left|f_{j}(\eta)-f_{j}(v)\right| \leq l_{j}|\eta-v|, \quad j \in \mathcal{N} .
$$

Let $\mathcal{S}=\mathcal{S}_{1} \times \mathcal{S}_{2} \times \cdots \times \mathcal{S}_{n}$, where $\mathcal{S}_{i}(i \in \mathcal{N})$ is the space consisting of continuous functions $\phi_{i}(t): \mathrm{R}_{+} \rightarrow \mathrm{R}$ such that $\phi_{i}(0)=x_{0 i}$ and $\phi_{i}(t) \rightarrow 0$ as $t \rightarrow \infty$, here $x_{0 i}$ is the same as defined in Section 2. Also $\mathcal{S}$ is a complete metric space when it is equipped with a metric defined by

$$
d(\overline{\mathbf{q}}(t), \overline{\mathbf{h}}(t))=\sup _{t \geq 0} \sum_{i=1}^{n}\left|q_{i}(t)-h_{i}(t)\right|
$$

where $\overline{\mathbf{q}}(t)=\left(q_{1}(t), \ldots, q_{n}(t)\right) \in \mathcal{S}$ and $\overline{\mathbf{h}}(t)=\left(h_{1}(t), \ldots, h_{n}(t)\right) \in \mathcal{S}$.

Theorem 3.1. Assume the condition (A1) holds. If the following inequalities hold

$$
\sum_{i=1}^{n}\left\{\frac{1}{a_{i}} \max _{j}\left\{\left|b_{i j} l_{j}\right|\right\}\right\}<1, \quad \max _{i \in \mathcal{N}}\left\{\lambda_{i}\right\}<\frac{1}{\sqrt{n}}
$$

where $\lambda_{i}=\left(1 / a_{i}\right) \sum_{j=1}^{n}\left|b_{i j} l_{j}\right|$, then the trivial equilibrium $\mathbf{x}=0$ of $(2.1)$ is asymptotically stable.

Proof. Multiplying both sides of (2.1) with $\mathrm{e}^{a_{i} t}$ gives

$$
\mathrm{de}^{a_{i} t} x_{i}(t)=\mathrm{e}^{a_{i} t} \mathrm{~d} x_{i}(t)+a_{i} x_{i}(t) \mathrm{e}^{a_{i} t} \mathrm{~d} t=\mathrm{e}^{a_{i} t} \sum_{j=1}^{n} b_{i j} f_{j}\left(x_{j}(t)\right) \mathrm{d} t, \quad t \geq 0, i \in \mathcal{N},
$$

which yields after integrating from 0 to $t$ as

$$
x_{i}(t)=x_{0 i} \mathrm{e}^{-a_{i} t}+\mathrm{e}^{-a_{i} t} \int_{0}^{t} \mathrm{e}^{a_{i} s} \sum_{j=1}^{n} b_{i j} f_{j}\left(x_{j}(s)\right) \mathrm{ds}, \quad t \geq 0, i \in \mathcal{N} .
$$

Now, for any $\overline{\mathbf{y}}(t)=\left(y_{1}(t), \ldots, y_{n}(t)\right) \in \mathcal{S}$, we define the following operator $\Phi$ acting on $\mathcal{S}$ as

$$
\Phi(\overline{\mathbf{y}})(t)=\left(\Phi\left(y_{1}\right)(t), \ldots, \Phi\left(y_{n}\right)(t)\right), \quad t \geq 0,
$$

where

$$
\Phi\left(y_{i}\right)(t)=x_{0 i} \mathrm{e}^{-a_{i} t}+\mathrm{e}^{-a_{i} t} \int_{0}^{t} \mathrm{e}^{a_{i} s} \sum_{j=1}^{n} b_{i j} f_{j}\left(y_{j}(s)\right) \mathrm{ds}, \quad i \in \mathcal{N} .
$$


The following proof is based on the contraction mapping principle, which can be divided into two steps as follows.

Step 1. We need to prove $\Phi(\mathcal{S}) \subset \mathcal{S}$. Recalling the construction of $\mathcal{S}$, we know that it is necessary to show the continuity of $\Phi$ on $[0, \infty)$ and $\left.\Phi\left(y_{i}\right)(t)\right|_{t=0}=x_{0 i}$ as well as $\lim _{t \rightarrow \infty} \Phi\left(y_{i}\right)(t)=0$ for $i \in \mathcal{N}$.

From (3.7), it is easy to see $\left.\Phi\left(y_{i}\right)(t)\right|_{t=0}=x_{0 i}$. Moreover, for a fixed time $t_{1} \geq 0$, we have

$$
\begin{aligned}
\Phi\left(y_{i}\right)\left(t_{1}+r\right)-\Phi\left(y_{i}\right)\left(t_{1}\right)= & x_{0 i} \mathrm{e}^{-a_{i}\left(t_{1}+r\right)} \\
& -x_{0 i} \mathrm{e}^{-a_{i} t_{1}}+\mathrm{e}^{-a_{i}\left(t_{1}+r\right)} \int_{0}^{t_{1}+r} \mathrm{e}^{a_{i} s} \sum_{j=1}^{n} b_{i j} f_{j}\left(y_{j}(s)\right) \mathrm{d} s \\
& -\mathrm{e}^{-a_{i} t_{1}} \int_{0}^{t_{1}} \mathrm{e}^{a_{i} s} \sum_{j=1}^{n} b_{i j} f_{j}\left(y_{j}(s)\right) \mathrm{ds} .
\end{aligned}
$$

It is not difficult to see that $\Phi\left(y_{i}\right)\left(t_{1}+r\right)-\Phi\left(y_{i}\right)\left(t_{1}\right) \rightarrow 0$ as $r \rightarrow 0$ which implies $\Phi$ is continuous on $[0, \infty)$.

Next we shall prove $\lim _{t \rightarrow \infty} \Phi\left(y_{i}\right)(t)=0$ for $y_{i}(t) \in S_{i}$. Since $y_{j}(t) \in \mathcal{S}_{j}$, we get $\lim _{t \rightarrow \infty} y_{j}(t)=0$. Then for any $\varepsilon>0$, there exists a $T_{j}>0$ such that $s \geq T_{j}$ implies $\left|y_{j}(s)\right|<\varepsilon$. Choose $T^{*}=\max _{j \in \mathcal{N}}\left\{T_{j}\right\}$. It is then derived form (A1) that

$$
\begin{aligned}
& \mathrm{e}^{-a_{i} t} \int_{0}^{t} \mathrm{e}^{a_{i} s} \sum_{j=1}^{n} b_{i j} f_{j}\left(y_{j}(s)\right) \mathrm{ds} \\
& \quad \leq \mathrm{e}^{-a_{i} t} \int_{0}^{t} \mathrm{e}^{a_{i} s} \sum_{j=1}^{n}\left\{\left|b_{i j} l_{j}\right|\left|y_{j}(s)\right|\right\} \mathrm{ds} \\
& \quad=\mathrm{e}^{-a_{i} t} \int_{0}^{T^{*}} \mathrm{e}^{a_{i} s} \sum_{j=1}^{n}\left\{\left|b_{i j} l_{j}\right|\left|y_{j}(s)\right|\right\} \mathrm{ds}+\mathrm{e}^{-a_{i} t} \int_{T^{*}}^{t} \mathrm{e}^{a_{i} s} \sum_{j=1}^{n}\left\{\left|b_{i j} l_{j}\right|\left|y_{j}(s)\right|\right\} \mathrm{ds} \\
& \quad \leq \mathrm{e}^{-a_{i} t} \sum_{j=1}^{n}\left\{\left|b_{i j} l_{j}\right| \sup _{s \in\left[0, T^{*}\right]}\left|y_{j}(s)\right|\right\}\left\{\int_{0}^{T^{*}} \mathrm{e}^{a_{i s} s} \mathrm{ds}\right\}+\varepsilon \mathrm{e}^{-a_{i} t} \sum_{j=1}^{n}\left\{\left|b_{i j} l_{j}\right|\right\} \int_{T^{*}}^{t} \mathrm{e}^{a_{i} s} \mathrm{ds} \\
& \quad \leq \mathrm{e}^{-a_{i} t} \sum_{j=1}^{n}\left\{\left|b_{i j} l_{j}\right| \sup _{s \in\left[0, T^{*}\right]}\left|y_{j}(s)\right|\right\}\left\{\int_{0}^{T^{*}} \mathrm{e}^{a_{i} s} \mathrm{ds}\right\}+\frac{\varepsilon}{a_{i}} \sum_{j=1}^{n}\left\{\left|b_{i j} l_{j}\right|\right\} .
\end{aligned}
$$

As $a_{i}>0$, we obtain $\mathrm{e}^{-a_{i} t} \int_{0}^{t} \mathrm{e}^{a_{i} s} \sum_{j=1}^{n} b_{i j} f_{j}\left(y_{j}(s)\right) \mathrm{d} s \rightarrow 0$ as $t \rightarrow \infty$. So $\lim _{t \rightarrow \infty} \Phi\left(y_{i}\right)(t)=0$ for $i \in \mathcal{N}$. We therefore conclude that $\Phi(\mathcal{S}) \subset \mathcal{S}$. 
Step 2. We need to prove $\Phi$ is contractive. For any $\overline{\mathbf{y}}=\left(y_{1}(t), \ldots, y_{n}(t)\right) \in \mathcal{S}$ and $\overline{\mathbf{z}}=$ $\left(z_{1}(t), \ldots, z_{n}(t)\right) \in \mathcal{S}$, we compute

$$
\begin{aligned}
& \sup _{t \in[0, T]} \sum_{i=1}^{n}\left|\Phi\left(y_{i}\right)(t)-\Phi\left(z_{i}\right)(t)\right| \\
& \quad \leq \sup _{t \in[0, T]} \sum_{i=1}^{n}\left\{\mathrm{e}^{-a_{i} t} \int_{0}^{t} \mathrm{e}^{a_{i} s} \sum_{j=1}^{n}\left\{\left|b_{i j}\right|\left|f_{j}\left(y_{j}(s)\right)-f_{j}\left(z_{j}(s)\right)\right|\right\} \mathrm{ds}\right\} \\
& \quad \leq \sup _{t \in[0, T]} \sum_{i=1}^{n}\left\{\mathrm{e}^{-a_{i} t} \int_{0}^{t} \mathrm{e}^{a_{i} s} \sum_{j=1}^{n}\left\{\left|b_{i j} l_{j}\right|\left|y_{j}(s)-z_{j}(s)\right|\right\} \mathrm{ds}\right\} \\
& \quad \leq \sup _{t \in[0, T]} \sum_{i=1}^{n}\left\{\max _{j \in \mathcal{N}}\left\{\left|b_{i j} l_{j}\right|\right\} \sup _{s \in[0, T]}\left\{\sum_{j=1}^{n}\left|y_{j}(s)-z_{j}(s)\right|\right\} \mathrm{e}^{-a_{i} t} \int_{0}^{t} \mathrm{e}^{a_{i} s} \mathrm{ds}\right\} \\
& \quad \leq \sum_{i=1}^{n}\left\{\frac{1}{a_{i}} \max _{j \in \mathcal{N}}\left\{\left|b_{i j} l_{j}\right|\right\}\right\} \sup _{s \in[0, T]}\left\{\sum_{j=1}^{n}\left|y_{j}(s)-z_{j}(s)\right|\right\} .
\end{aligned}
$$

As $\sum_{i=1}^{n}\left\{\left(1 / a_{i}\right) \max _{j \in \mathcal{N}}\left\{\left|b_{i j} l_{j}\right|\right\}\right\}<1, \Phi$ is a contraction mapping.

Therefore, by the contraction mapping principle, we see there must exist a unique fixed point $\overline{\mathbf{u}}(\cdot)$ of $\Phi$ in $\mathcal{S}$ which means $\overline{\mathbf{u}}^{\mathrm{T}}(\cdot)$ is the solution of (2.1)-(2.2) and $\left\|\overline{\mathbf{u}}^{\mathrm{T}}(\cdot)\right\| \rightarrow 0$ as $t \rightarrow \infty$.

To obtain the asymptotic stability, we still need to prove that the trivial equilibrium $\mathbf{x}=0$ of (2.1) is stable. For any $\varepsilon>0$, from the conditions of Theorem 3.1, we can find $\delta$ satisfying $0<\delta<\varepsilon$ such that $\delta+\max _{i \in \mathcal{N}}\left\{\lambda_{i}\right\} \varepsilon \leq \varepsilon / \sqrt{n}$.

Let $\left\|\mathbf{x}_{0}\right\|<\delta$. According to what have been discussed above, we know that there must exist a unique solution $\mathbf{x}\left(t ; 0, \mathbf{x}_{0}\right)=\left(x_{1}\left(t ; 0, x_{01}\right), \ldots, x_{n}\left(t ; 0, x_{0 n}\right)\right)^{\mathrm{T}} \in \mathrm{R}^{n}$ to $(2.1)-(2.2)$, and

$$
x_{i}(t)=\Phi\left(x_{i}\right)(t)=J_{1}(t)+J_{2}(t), \quad t \geq 0,
$$

where $J_{1}(t)=x_{0 i} \mathrm{e}^{-a_{i} t}, J_{2}(t)=\mathrm{e}^{-a_{i} t} \int_{0}^{t} \mathrm{e}^{a_{i} s} \sum_{j=1}^{n} b_{i j} f_{j}\left(x_{j}(s)\right) \mathrm{ds}$.

Suppose there exists $t^{*}>0$ such that $\left\|\mathbf{x}\left(t^{*} ; 0, \mathbf{x}_{0}\right)\right\|=\varepsilon$ and $\left\|\mathbf{x}\left(t ; 0, \mathbf{x}_{0}\right)\right\|<\varepsilon$ as $0 \leq t<t^{*}$. It follows from (3.11) that $\left|x_{i}\left(t^{*}\right)\right| \leq\left|J_{1}\left(t^{*}\right)\right|+\left|J_{2}\left(t^{*}\right)\right|$.

As $\left|J_{1}\left(t^{*}\right)\right|=\left|x_{0 i} \mathrm{e}^{-a_{i} t^{*}}\right| \leq \delta$ and $\left|J_{2}\left(t^{*}\right)\right| \leq \mathrm{e}^{-a_{i} t^{*}} \int_{0}^{t^{*}} \mathrm{e}^{a_{i} s} \sum_{j=1}^{n}\left|b_{i j} l_{j} x_{j}(s)\right| \mathrm{ds}<$ $\left(\varepsilon / a_{i}\right) \sum_{j=1}^{n}\left|b_{i j} l_{j}\right|$, we obtain $\left|x_{i}\left(t^{*}\right)\right|<\delta+\lambda_{i} \varepsilon$. Hence

$$
\left\|\mathbf{x}\left(t^{*} ; 0, \mathbf{x}_{0}\right)\right\|^{2}=\sum_{i=1}^{n}\left\{\left|x_{i}\left(t^{*}\right)\right|^{2}\right\}<\sum_{i=1}^{n}\left\{\left|\delta+\lambda_{i} \varepsilon\right|^{2}\right\} \leq n\left|\delta+\max _{i \in \mathcal{N}}\left\{\lambda_{i}\right\} \varepsilon\right|^{2} \leq \varepsilon^{2} .
$$

This contradicts to the assumption of $\left\|\mathbf{x}\left(t^{*} ; 0, \mathbf{x}_{0}\right)\right\|=\varepsilon$. Therefore, $\left\|\mathbf{x}\left(t ; 0, \mathbf{x}_{0}\right)\right\|<\varepsilon$ holds for all $t \geq 0$. This completes the proof. 


\section{Asymptotic Stability of Delayed Cellular Neural Networks}

In this section, we will simultaneously consider the existence and uniqueness of solution to (2.3)-(2.4) and the asymptotic stability of trivial equilibrium $\mathbf{x}=0$ of (2.3) by means of the contraction mapping principle. Before proceeding, we give the assumption as follows.

(A2) There exist nonnegative constants $k_{j}$ such that for $\eta, v \in \mathrm{R}$,

$$
\left|g_{j}(\eta)-g_{j}(v)\right| \leq k_{j}|\eta-v|, \quad j \in \mathcal{N} .
$$

Let $\mathscr{H}=\mathscr{L}_{1} \times \cdots \times \mathscr{H}_{n}$, where $\mathscr{H}_{i}(i \in \mathcal{N})$ is the space consisting of continuous functions $\phi_{i}(t):[-\tau, \infty) \rightarrow \mathrm{R}$ such that $\phi_{i}(s)=\varphi_{i}(s)$ on $s \in[-\tau, 0]$ and $\phi_{i}(t) \rightarrow 0$ as $t \rightarrow \infty$, here $\varphi_{i}(s)$ is the same as defined in Section 2. Also $\mathscr{H}$ is a complete metric space when it is equipped with a metric defined by

$$
d(\overline{\mathbf{q}}(t), \overline{\mathbf{h}}(t))=\sup _{t \geq-\tau} \sum_{i=1}^{n}\left|q_{i}(t)-h_{i}(t)\right|
$$

where $\overline{\mathbf{q}}(t)=\left(q_{1}(t), \ldots, q_{n}(t)\right) \in \mathscr{H}$ and $\overline{\mathbf{h}}(t)=\left(h_{1}(t), \ldots, h_{n}(t)\right) \in \mathscr{H}$.

Theorem 4.1. Assume the conditions (A1)-(A2) hold. If the following inequalities hold

$$
\sum_{i=1}^{n}\left\{\frac{1}{a_{i}}\left(\max _{j \in \mathcal{N}}\left|b_{i j} l_{j}\right|+\max _{j \in \mathcal{N}}\left|c_{i j} k_{j}\right|\right)\right\}<1, \quad \max _{i \in \mathcal{N}}\left\{\lambda_{i}^{*}\right\}<\frac{1}{\sqrt{n}}
$$

where $\lambda_{i}{ }^{*}=\left(1 / a_{i}\right) \sum_{j=1}^{n}\left|b_{i j} l_{j}\right|+\left(1 / a_{i}\right) \sum_{j=1}^{n}\left|c_{i j} k_{j}\right|$, then the trivial equilibrium $\mathbf{x}=0$ of (2.3) is asymptotically stable.

Proof. Multiplying both sides of (2.3) with $\mathrm{e}^{a_{i} t}$ gives

$$
\begin{aligned}
\mathrm{de}^{a_{i} t} x_{i}(t) & =\mathrm{e}^{a_{i} t} \mathrm{~d} x_{i}(t)+a_{i} x_{i}(t) \mathrm{e}^{a_{i} t} \mathrm{~d} t \\
& =\mathrm{e}^{a_{i} t}\left\{\sum_{j=1}^{n} b_{i j} f_{j}\left(x_{j}(t)\right)+\sum_{j=1}^{n} c_{i j} g_{j}\left(x_{j}\left(t-\tau_{j}(t)\right)\right)\right\} \mathrm{d} t,
\end{aligned}
$$

which yields after integrating from 0 to $t$ as

$$
x_{i}(t)=\varphi_{i}(0) \mathrm{e}^{-a_{i} t}+\mathrm{e}^{-a_{i} t} \int_{0}^{t} \mathrm{e}^{a_{i} s}\left\{\sum_{j=1}^{n} b_{i j} f_{j}\left(x_{j}(s)\right)+\sum_{j=1}^{n} c_{i j} g_{j}\left(x_{j}\left(s-\tau_{j}(s)\right)\right)\right\} \mathrm{d} s .
$$

Now for any $\overline{\mathbf{y}}(t)=\left(y_{1}(t), \ldots, y_{n}(t)\right) \in \mathscr{L}$, we define the following operator $\pi$ acting on $\mathscr{d}$

$$
\pi(\overline{\mathbf{y}})(t)=\left(\pi\left(y_{1}\right)(t), \ldots, \pi\left(y_{n}\right)(t)\right),
$$


where

$$
\pi\left(y_{i}\right)(t)=\varphi_{i}(0) \mathrm{e}^{-a_{i} t}+\mathrm{e}^{-a_{i} t} \int_{0}^{t} \mathrm{e}^{a_{i} s}\left\{\sum_{j=1}^{n} b_{i j} f_{j}\left(y_{j}(s)\right)+\sum_{j=1}^{n} c_{i j} g_{j}\left(y_{j}\left(s-\tau_{j}(s)\right)\right)\right\} \mathrm{ds}, \quad t \geq 0,
$$

and $\pi\left(y_{i}\right)(s)=\varphi_{i}(s)$ on $s \in[-\tau, 0]$ for $i \in \mathcal{N}$.

Similar to the proof of Theorem 3.1, we shall apply the contraction mapping principle to prove Theorem 4.1. The subsequent proof can be divided into two steps.

Step 1 . We need prove $\pi(\mathscr{l}) \subset \mathscr{d}$. To prove $\pi(\mathscr{l}) \subset \mathscr{d}$, it is necessary to show the continuity of $\pi$ on $[-\tau, \infty)$ and $\lim _{t \rightarrow \infty} \pi\left(y_{i}\right)(t)=0$ for $y_{i}(t) \in \mathscr{L}_{i}$ and $i \in \mathcal{N}$. In light of (4.7), we have, for a fixed time $t_{1} \geq 0$,

$$
\pi\left(y_{i}\right)\left(t_{1}+r\right)-\pi\left(y_{i}\right)\left(t_{1}\right)=I_{1}+I_{2}+I_{3}
$$

where

$$
\begin{aligned}
& I_{1}=\varphi_{i}(0) \mathrm{e}^{-a_{i}\left(t_{1}+r\right)}-\varphi_{i}(0) \mathrm{e}^{-a_{i} t_{1}}, \\
& I_{2}=\mathrm{e}^{-a_{i}\left(t_{1}+r\right)} \int_{0}^{t_{1}+r} \mathrm{e}^{a_{i} s} \sum_{j=1}^{n} b_{i j} f_{j}\left(y_{j}(s)\right) \mathrm{ds}-\mathrm{e}^{-a_{i} t_{1}} \int_{0}^{t_{1}} \mathrm{e}^{a_{i} s} \sum_{j=1}^{n} b_{i j} f_{j}\left(y_{j}(s)\right) \mathrm{ds}, \\
& I_{3}=\mathrm{e}^{-a_{i}\left(t_{1}+r\right)} \int_{0}^{t_{1}+r} \mathrm{e}^{a_{i} s} \sum_{j=1}^{n} c_{i j} g_{j}\left(y_{j}\left(s-\tau_{j}(s)\right)\right) \mathrm{d} s-\mathrm{e}^{-a_{i} t_{1}} \int_{0}^{t_{1}} \mathrm{e}^{a_{i} s} \sum_{j=1}^{n} c_{i j} g_{j}\left(y_{j}\left(s-\tau_{j}(s)\right)\right) \mathrm{d} \mathrm{s} .
\end{aligned}
$$

It is easy to see that $\lim _{r \rightarrow 0}\left\{\pi\left(y_{i}\right)\left(t_{1}+r\right)-\pi\left(y_{i}\right)\left(t_{1}\right)\right\}=0$. Thus, $\pi$ is continuous on $[0, \infty)$. Noting $\varphi_{i}(s) \in C([-\tau, 0], R)$ and $\pi\left(y_{i}\right)(0)=\varphi_{i}(0)$, we obtain $\pi$ is indeed continuous on $[-\tau, \infty)$.

Next, we will prove $\lim _{t \rightarrow \infty} \pi\left(y_{i}\right)(t)=0$ for $y_{i}(t) \in \mathscr{H}_{i}$. As we did in Section 3, we know $\lim _{t \rightarrow \infty} \mathrm{e}^{-a_{i} t}=0$ and $\mathrm{e}^{-a_{i} t} \int_{0}^{t} \mathrm{e}^{a_{i} s} \sum_{j=1}^{n} b_{i j} f_{j}\left(y_{j}(s)\right) \mathrm{ds} \rightarrow 0$ as $t \rightarrow \infty$. In what follows, we will show $\mathrm{e}^{-a_{i} t} \int_{0}^{t} \mathrm{e}^{a_{i} s} \sum_{j=1}^{n} c_{i j} g_{j}\left(y_{j}\left(s-\tau_{j}(s)\right)\right) \mathrm{ds} \rightarrow 0$ as $t \rightarrow \infty$. In fact, since $y_{j}(t) \in \mathscr{L}_{j}$, we have $\lim _{t \rightarrow \infty} y_{j}(t)=0$. Then for any $\varepsilon>0$, there exists a $T_{j}{ }^{\prime}>0$ such that $s \geq T_{j}{ }^{\prime}-\tau$ implies $\left|y_{j}(s)\right|<\varepsilon$. Select $\widehat{T}=\max _{j \in \mathcal{N}}\left\{T_{j}{ }^{\prime}\right\}$. It is then derived from (A2) that

$$
\begin{aligned}
& \mathrm{e}^{-a_{i} t} \int_{0}^{t} \mathrm{e}^{a_{i} s} \sum_{j=1}^{n} c_{i j} g_{j}\left(y_{j}\left(s-\tau_{j}(s)\right)\right) \mathrm{ds} \\
& \leq \mathrm{e}^{-a_{i} t} \int_{0}^{t} \mathrm{e}^{a_{i} s} \sum_{j=1}^{n}\left\{\left|c_{i j} k_{j}\right|\left|y_{j}\left(s-\tau_{j}(s)\right)\right|\right\} \mathrm{ds} \\
& =\mathrm{e}^{-a_{i} t} \int_{0}^{\widehat{T}} \mathrm{e}^{a_{i} s} \sum_{j=1}^{n}\left\{\left|c_{i j} k_{j}\right|\left|y_{j}\left(s-\tau_{j}(s)\right)\right|\right\} \mathrm{ds} \\
& \quad+\mathrm{e}^{-a_{i} t} \int_{\widehat{T}}^{t} \mathrm{e}^{a_{i} s} \sum_{j=1}^{n}\left\{\left|c_{i j} k_{j}\right|\left|y_{j}\left(s-\tau_{j}(s)\right)\right|\right\} \mathrm{ds}
\end{aligned}
$$


Journal of Applied Mathematics

$$
\begin{aligned}
& \leq \mathrm{e}^{-a_{i} t} \sum_{j=1}^{n}\left\{\left|c_{i j} k_{j}\right| \sup _{s \in[-\tau, \widehat{T}]}\left|y_{j}(s)\right|\right\}\left\{\int_{0}^{\widehat{T}} \mathrm{e}^{\mathrm{a}_{\mathrm{i}} \mathrm{s}} \mathrm{ds}\right\}+\varepsilon \mathrm{e}^{-a_{i} t} \sum_{j=1}^{n}\left\{\left|c_{i j} k_{j}\right|\right\} \int_{\widehat{T}}^{t} \mathrm{e}^{a_{i} s} \mathrm{ds} \\
& \leq \mathrm{e}^{-a_{i} t} \sum_{j=1}^{n}\left\{\left|c_{i j} k_{j}\right| \sup _{s \in[-\tau, \widehat{T}]}\left|y_{j}(s)\right|\right\}\left\{\int_{0}^{\widehat{T}} \mathrm{e}^{a_{i} s} \mathrm{ds}\right\}+\frac{\varepsilon}{a_{i}} \sum_{j=1}^{n}\left\{\left|c_{i j} k_{j}\right|\right\} .
\end{aligned}
$$

As $\lim _{t \rightarrow \infty} \mathrm{e}^{-a_{i} t}=0$, we obtain $\mathrm{e}^{-a_{i} t} \int_{0}^{t} \mathrm{e}^{a_{i} s} \sum_{j=1}^{n} c_{i j} g_{j}\left(y_{j}\left(s-\tau_{j}(s)\right)\right) \mathrm{d} s \rightarrow 0$ as $t \rightarrow \infty$, which leads to $\lim _{t \rightarrow \infty} \pi\left(y_{i}\right)(t)=0$ for $y_{i}(t) \in \mathscr{\ell}_{i}$ and $i \in \mathcal{N}$. We therefore conclude $\pi(\mathscr{t}) \subset \mathscr{H}$.

Step 2. We need to prove $\pi$ is contractive. For any $\overline{\mathbf{y}}=\left(y_{1}(t), \ldots, y_{n}(t)\right) \in \mathscr{H}$ and $\overline{\mathbf{z}}=$ $\left(z_{1}(t), \ldots, z_{n}(t)\right) \in \mathscr{\ell}$, we estimate

$$
\begin{aligned}
& \sum_{i=1}^{n}\left|\pi\left(y_{i}\right)(t)-\pi\left(z_{i}\right)(t)\right| \\
& \leq \sum_{i=1}^{n}\left\{\mathrm{e}^{-a_{i} t} \int_{0}^{t} \mathrm{e}^{a_{i} s} \sum_{j=1}^{n}\left[\left|b_{i j}\right|\left|f_{j}\left(y_{j}(s)\right)-f_{j}\left(z_{j}(s)\right)\right|\right] \mathrm{ds}\right\} \\
& +\sum_{i=1}^{n}\left\{\mathrm{e}^{-a_{i} t} \int_{0}^{t} \mathrm{e}^{a_{i} s} \sum_{j=1}^{n}\left[\left|c_{i j}\right|\left|g_{j}\left(y_{j}\left(s-\tau_{j}(s)\right)\right)-g_{j}\left(z_{j}\left(s-\tau_{j}(s)\right)\right)\right|\right] \mathrm{d} s\right\} \\
& \leq \sum_{i=1}^{n}\left\{\mathrm{e}^{-a_{i} t} \int_{0}^{t} \mathrm{e}^{a_{i} s} \sum_{j=1}^{n}\left[\left|b_{i j} l_{j}\right|\left|y_{j}(s)-z_{j}(s)\right|\right] \mathrm{ds}\right\} \\
& +\sum_{i=1}^{n}\left\{\mathrm{e}^{-a_{i} t} \int_{0}^{t} \mathrm{e}^{a_{i} s} \sum_{j=1}^{n}\left[\left|c_{i j} k_{j}\right|\left|y_{j}\left(s-\tau_{j}(s)\right)-z_{j}\left(s-\tau_{j}(s)\right)\right|\right] \mathrm{d} s\right\} \\
& \leq \sum_{i=1}^{n}\left\{\max _{j \in \mathcal{N}}\left|b_{i j} l_{j}\right| \sup _{s \in[0, t]}\left\{\sum_{j=1}^{n}\left|y_{j}(s)-z_{j}(s)\right|\right\} e^{-a_{i} t} \int_{0}^{t} e^{a_{i} s} \mathrm{ds}\right\} \\
& +\sum_{i=1}^{n}\left\{\max _{j \in \mathcal{N}}\left|c_{i j} k_{j}\right| \sup _{s \in[-\tau, t]}\left\{\sum_{j=1}^{n}\left|y_{j}(s)-z_{j}(s)\right|\right\} \mathrm{e}^{-a_{i} t} \int_{0}^{t} \mathrm{e}^{a_{i} s} \mathrm{ds}\right\} \\
& \leq \sum_{i=1}^{n}\left\{\frac{1}{a_{i}} \max _{j \in \mathcal{N}}\left|b_{i j} l_{j}\right|\right\} \sup _{s \in[0, t]}\left\{\sum_{j=1}^{n}\left|y_{j}(s)-z_{j}(s)\right|\right\} \\
& +\sum_{i=1}^{n}\left\{\frac{1}{a_{i}} \max _{j \in \mathcal{N}}\left|c_{i j} k_{j}\right|\right\} \sup _{s \in[-\tau, t]}\left\{\sum_{j=1}^{n}\left|y_{j}(s)-z_{j}(s)\right|\right\} .
\end{aligned}
$$


Hence,

$$
\begin{aligned}
& \sup _{t \in[-\tau, T]} \sum_{i=1}^{n}\left|\pi\left(y_{i}\right)(t)-\pi\left(z_{i}\right)(t)\right| \\
& \quad \leq\left\{\sum_{i=1}^{n}\left\{\frac{1}{a_{i}} \max _{j \in \mathcal{N}}\left|b_{i j} l_{j}\right|\right\}+\sum_{i=1}^{n}\left\{\frac{1}{a_{i}} \max _{j \in \mathcal{N}}\left|c_{i j} k_{j}\right|\right\}\right\} \sup _{s \in[-\tau, T]}\left\{\sum_{j=1}^{n}\left|y_{j}(s)-z_{j}(s)\right|\right\} .
\end{aligned}
$$

As $\sum_{i=1}^{n}\left\{\left(1 / a_{i}\right)\left(\max _{j \in \mathcal{N}}\left|b_{i j} l_{j}\right|+\max _{j \in \mathcal{N}}\left|c_{i j} k_{j}\right|\right)\right\}<1, \pi$ is a contraction mapping and hence there exists a unique fixed point $\overline{\mathbf{u}}(\cdot)$ of $\pi$ in $\mathscr{t}$ which means $\overline{\mathbf{u}}^{\mathrm{T}}(\cdot)$ is the solution of (2.3)-(2.4) and $\left\|\overline{\mathbf{u}}^{\mathrm{T}}(\cdot)\right\| \rightarrow 0$ as $t \rightarrow \infty$.

To obtain the asymptotic stability, we still need to prove that the trivial equilibrium of (2.3) is stable. For any $\varepsilon>0$, from the conditions of Theorem 4.1, we can find $\delta$ satisfying $0<\delta<\varepsilon$ such that $\delta+\max _{i \in \mathcal{N}}\left\{\lambda_{i}^{*}\right\} \varepsilon \leq \varepsilon / \sqrt{n}$.

Let $|\varphi|<\delta$. According to what have been discussed above, we know that there exists a unique solution $\mathbf{x}(t ; s, \varphi)=\left(x_{1}\left(t ; s, \varphi_{1}\right), \ldots, x_{n}\left(t ; s, \varphi_{n}\right)\right)^{\mathrm{T}} \in \mathrm{R}^{n}$ to $(2.3)-(2.4)$, and

$$
x_{i}(t)=\pi\left(x_{i}\right)(t)=J_{1}(t)+J_{2}(t)+J_{3}(t), \quad t \geq 0,
$$

where

$$
\begin{aligned}
& J_{1}(t)=x_{0 i} \mathrm{e}^{-a_{i} t}, \quad J_{2}(t)=\mathrm{e}^{-a_{i} t} \int_{0}^{t} \mathrm{e}^{a_{i} s} \sum_{j=1}^{n} b_{i j} f_{j}\left(x_{j}(s)\right) \mathrm{ds}, \\
& J_{3}=\mathrm{e}^{-a_{i} t} \int_{0}^{t} \mathrm{e}^{a_{i} s} \sum_{j=1}^{n} c_{i j} g_{j}\left(x_{j}\left(s-\tau_{j}(s)\right)\right) \mathrm{ds} .
\end{aligned}
$$

Suppose there exists $t^{*}>0$ such that $\left\|\mathbf{x}\left(t^{*} ; s, \varphi\right)\right\|=\varepsilon$ and $\|\mathbf{x}(t ; s, \varphi)\|<\varepsilon$ as $0 \leq t<t^{*}$. It follows from (4.13) that $\left|x_{i}\left(t^{*}\right)\right| \leq\left|J_{1}\left(t^{*}\right)\right|+\left|J_{2}\left(t^{*}\right)\right|+\left|J_{3}\left(t^{*}\right)\right|$.

$$
\begin{aligned}
& \text { As }\left|J_{1}\left(t^{*}\right)\right|=\left|x_{0 i} \mathrm{e}^{-a_{i} t^{*}}\right| \leq \delta,\left|J_{2}\left(t^{*}\right)\right|<\left(\varepsilon / a_{i}\right) \sum_{j=1}^{n}\left|b_{i j} l_{j}\right| \text { and } \\
& \left|J_{3}\left(t^{*}\right)\right| \leq \mathrm{e}^{-a_{i} t^{*}} \int_{0}^{t^{*}} \mathrm{e}^{a_{i} s} \sum_{j=1}^{n}\left|c_{i j} k_{j} x_{j}\left(s-\tau_{j}(s)\right)\right| \mathrm{ds}<\frac{\varepsilon}{a_{i}} \sum_{j=1}^{n}\left|c_{i j} k_{j}\right|,
\end{aligned}
$$

we obtain $\left|x_{i}\left(t^{*}\right)\right|<\delta+\lambda_{i}^{*} \varepsilon$. Hence

$$
\left\|\mathbf{x}\left(t^{*} ; s, \varphi\right)\right\|^{2}=\sum_{i=1}^{n}\left\{\left|x_{i}\left(t^{*}\right)\right|^{2}\right\}<\sum_{i=1}^{n}\left\{\left|\delta+\lambda_{i}^{*} \varepsilon\right|^{2}\right\} \leq n\left|\delta+\max _{i \in \mathcal{N}}\left\{\lambda_{i}^{*}\right\} \varepsilon\right|^{2} \leq \varepsilon^{2} .
$$

This contradicts to the assumption of $\left\|\mathbf{x}\left(t^{*} ; s, \varphi\right)\right\|=\varepsilon$. Therefore, $\|\mathbf{x}(t ; s, \varphi)\|<\varepsilon$ holds for all $t \geq 0$. This completes the proof.

Remark 4.2. In Theorems 3.1 and 4.1, we use the contraction mapping principle to study the existence and uniqueness of solution and the asymptotic stability of trivial equilibrium at the same time, while Lyapunov method fails to do this. 
Remark 4.3. The provided sufficient conditions in Theorem 4.1 do not require even the differentiability of delays, let alone the monotone decreasing behavior of delays which is necessary in some relevant works.

\section{Example}

Consider the following two-dimensional cellular neural network with time-varying delays

$$
\frac{\mathrm{d} x_{i}(t)}{\mathrm{d} t}=-a_{i} x_{i}(t)+\sum_{j=1}^{2} b_{i j} f_{j}\left(x_{j}(t)\right)+\sum_{j=1}^{2} c_{i j} g_{j}\left(x_{j}\left(t-\tau_{j}(t)\right)\right),
$$

with the initial conditions $x_{1}(s)=\cos (s), x_{2}(s)=\sin (s)$ on $-\tau \leq s \leq 0$, where $a_{1}=a_{2}=$ $3, b_{11}=0, b_{12}=1 / 7, b_{21}=-1 / 7, b_{22}=-1 / 7, c_{11}=3 / 7, c_{12}=2 / 7, c_{21}=0, c_{22}=1 / 7$, $f_{j}(s)=g_{j}(s)=(|s+1|-|s-1|) / 2, \tau_{j}(t)$ is bounded by $\tau$.

It is easily to know that $l_{j}=k_{j}=1$ for $j=1,2$. Compute

$$
\sum_{i=1}^{2}\left\{\frac{1}{a_{i}}\left(\max _{j=1,2}\left|b_{i j} l_{j}\right|+\max _{j=1,2}\left|c_{i j} k_{j}\right|\right)\right\}<1, \quad \max _{i \in \mathcal{N}}\left\{\frac{1}{a_{i}} \sum_{j=1}^{n}\left|b_{i j} l_{j}\right|+\frac{1}{a_{i}} \sum_{j=1}^{n}\left|c_{i j} k_{j}\right|\right\}<\frac{1}{\sqrt{2}} .
$$

From Theorem 4.1, we conclude that the trivial equilibrium $\mathbf{x}=0$ of this two-dimensional cellular neural network is asymptotically stable.

\section{Acknowledgments}

This work is supported by National Natural Science Foundation of China under Grant 60904028 and 71171116.

\section{References}

[1] L. O. Chua and L. Yang, "Cellular neural networks: theory," Institute of Electrical and Electronics Engineers, vol. 35, no. 10, pp. 1257-1272, 1988.

[2] L. O. Chua and L. Yang, "Cellular neural networks: applications," Institute of Electrical and Electronics Engineers, vol. 35, no. 10, pp. 1273-1290, 1988.

[3] J. Cao, "New results concerning exponential stability and periodic solutions of delayed cellular neural networks," Physics Letters A, vol. 307, no. 2-3, pp. 136-147, 2003.

[4] P. P. Civalleri, M. Gilli, and L. Pandolfi, “On stability of cellular neural networks with delay," IEEE Transactions on Circuits and Systems, vol. 40, no. 3, pp. 157-165, 1993.

[5] J. Cao, "A set of stability criteria for delayed cellular neural networks," IEEE Transactions on Circuits and Systems, vol. 48, no. 4, pp. 494-498, 2001.

[6] G. T. Stamov and I. M. Stamova, "Almost periodic solutions for impulsive neural networks with delay," Applied Mathematical Modelling, vol. 31, pp. 1263-1270, 2007.

[7] S. Ahmad and I. M. Stamova, "Global exponential stability for impulsive cellular neural networks with time-varying delays," Nonlinear Analysis, vol. 69, no. 3, pp. 786-795, 2008.

[8] K. Li, X. Zhang, and Z. Li, "Global exponential stability of impulsive cellular neural networks with time-varying and distributed delay," Chaos, Solitons and Fractals, vol. 41, no. 3, pp. 1427-1434, 2009.

[9] J. Qiu, "Exponential stability of impulsive neural networks with time-varying delays and reactiondiffusion terms," Neurocomputing, vol. 70, pp. 1102-1108, 2007. 
[10] X. Wang and D. Xu, "Global exponential stability of impulsive fuzzy cellular neural networks with mixed delays and reaction-diffusion terms," Chaos, Solitons E Fractals, vol. 42, no. 5, pp. 2713-2721, 2009.

[11] Y. Zhang and Q. Luo, "Global exponential stability of impulsive delayed reaction-diffusion neural networks via Hardy-Poincarè Inequality," Neurocomputing, vol. 83, pp. 198-204, 2012.

[12] T. A. Burton, Stability by Fixed Point Theory for Functional Differential Equations, Dover, New York, NY, USA, 2006.

[13] L. C. Becker and T. A. Burton, "Stability, fixed points and inverses of delays," Proceedings of the Royal Society of Edinburgh A, vol. 136, no. 2, pp. 245-275, 2006.

[14] T. A. Burton, "Fixed points, stability, and exact linearization," Nonlinear Analysis, vol. 61, no. 5, pp. 857-870, 2005.

[15] T. A. Burton, "Fixed points, Volterra equations, and Becker's resolvent," Acta Mathematica Hungarica, vol. 108, no. 3, pp. 261-281, 2005.

[16] T. A. Burton, "Fixed points and stability of a nonconvolution equation," Proceedings of the American Mathematical Society, vol. 132, no. 12, pp. 3679-3687, 2004.

[17] T. A. Burton, "Perron-type stability theorems for neutral equations," Nonlinear Analysis, vol. 55, no. 3, pp. 285-297, 2003.

[18] T. A. Burton, "Integral equations, implicit functions, and fixed points," Proceedings of the American Mathematical Society, vol. 124, no. 8, pp. 2383-2390, 1996.

[19] T. A. Burton and T. Furumochi, "Krasnoselskii's fixed point theorem and stability," Nonlinear Analysis, vol. 49, no. 4, pp. 445-454, 2002.

[20] T. A. Burton and B. Zhang, "Fixed points and stability of an integral equation: nonuniqueness," Applied Mathematics Letters, vol. 17, no. 7, pp. 839-846, 2004.

[21] T. Furumochi, "Stabilities in FDEs by Schauder's theorem," Nonlinear Analysis, vol. 63, pp. 217-224, 2005.

[22] C. Jin and J. Luo, "Fixed points and stability in neutral differential equations with variable delays," Proceedings of the American Mathematical Society, vol. 136, no. 3, pp. 909-918, 2008.

[23] Y. N. Raffoul, "Stability in neutral nonlinear differential equations with functional delays using fixedpoint theory," Mathematical and Computer Modelling, vol. 40, no. 7-8, pp. 691-700, 2004.

[24] B. Zhang, "Fixed points and stability in differential equations with variable delays," Nonlinear Analysis, vol. 63, pp. 233-242, 2005.

[25] J. Luo, "Fixed points and stability of neutral stochastic delay differential equations," Journal of Mathematical Analysis and Applications, vol. 334, no. 1, pp. 431-440, 2007.

[26] J. Luo, "Fixed points and exponential stability of mild solutions of stochastic partial differential equations with delays," Journal of Mathematical Analysis and Applications, vol. 342, no. 2, pp. 753-760, 2008.

[27] J. Luo, "Stability of stochastic partial differential equations with infinite delays," Journal of Computational and Applied Mathematics, vol. 222, no. 2, pp. 364-371, 2008.

[28] J. Luo and T. Taniguchi, "Fixed points and stability of stochastic neutral partial differential equations with infinite delays," Stochastic Analysis and Applications, vol. 27, no. 6, pp. 1163-1173, 2009.

[29] R. Sakthivel and J. Luo, "Asymptotic stability of impulsive stochastic partial differential equations with infinite delays," Journal of Mathematical Analysis and Applications, vol. 356, no. 1, pp. 1-6, 2009.

[30] R. Sakthivel and J. Luo, "Asymptotic stability of nonlinear impulsive stochastic differential equations," Statistics E Probability Letters, vol. 79, no. 9, pp. 1219-1223, 2009.

[31] J. Luo, "Fixed points and exponential stability for stochastic Volterra-Levin equations," Journal of Computational and Applied Mathematics, vol. 234, no. 3, pp. 934-940, 2010.

[32] X. X. Liao, Theory and Application of Stability for Dynamical Systems, National Defence Industry Press, 2000.

[33] D. R. Smart, Fixed Point Theorems, Cambridge University Press, Cambridge, UK, 1980. 


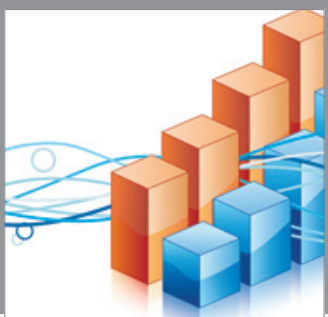

Advances in

Operations Research

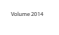

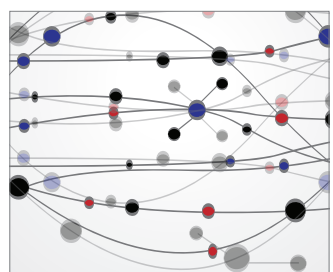

\section{The Scientific} World Journal
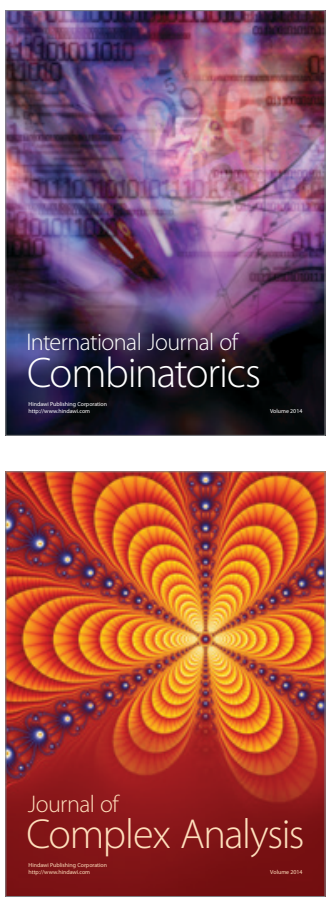

International Journal of

Mathematics and

Mathematical

Sciences
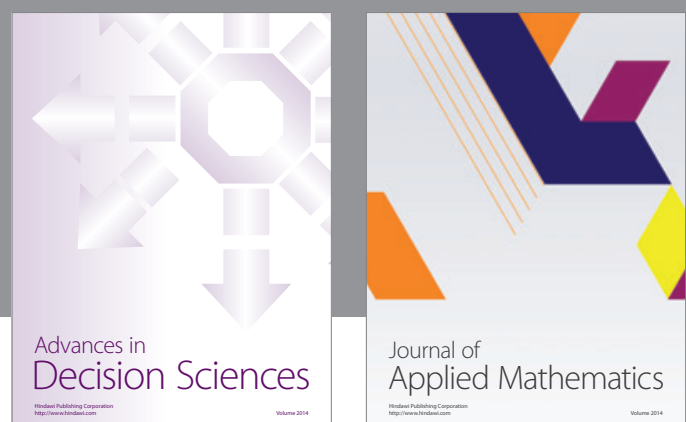

Journal of

Applied Mathematics
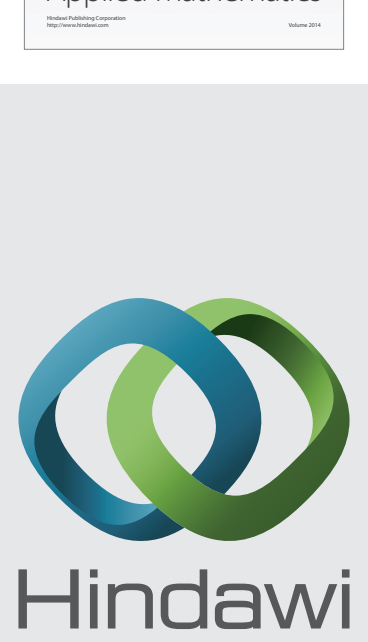

Submit your manuscripts at http://www.hindawi.com
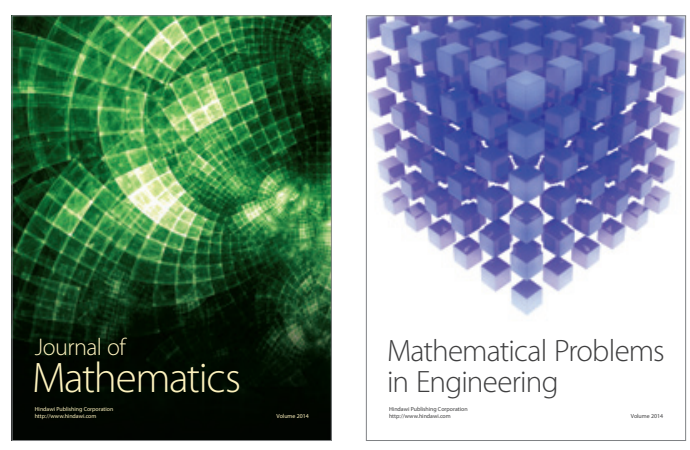

Mathematical Problems in Engineering
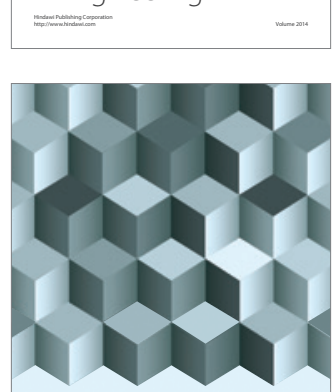

Journal of

Function Spaces
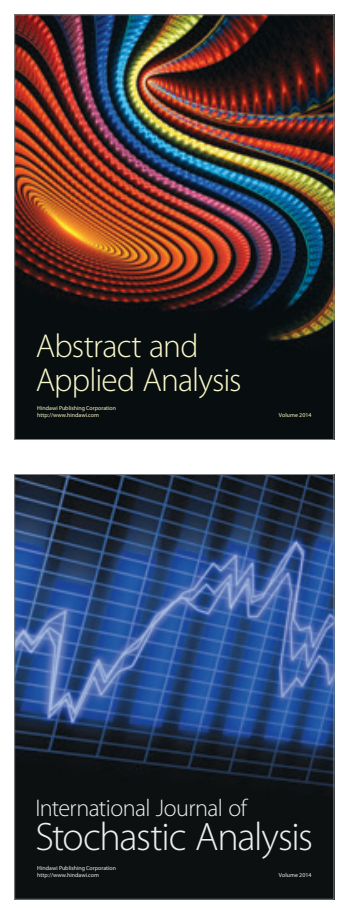

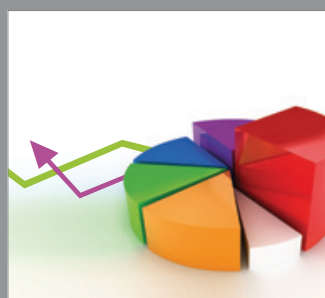

ournal of

Probability and Statistics

Promensencen
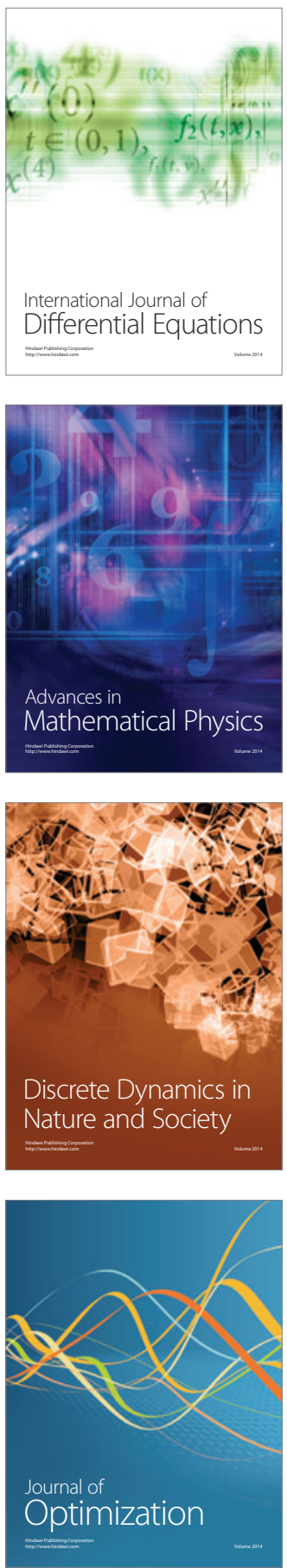